OPEN

SUBJECT AREAS:

X-RAY

CRYSTALLOGRAPHY

STRUCTURAL BIOLOGY

Received

7 July 2014

Accepted

17 November 2014

Published

3 December 2014

Correspondence and requests for materials should be addressed to

T.T. (ttsukaza@bs. naist.jp) or O.N. (nureki@bs.s.u-tokyo.

ac.jp)

\section{Crystal structure of Escherichia coli YidC, a membrane protein chaperone and} insertase

\author{
Kaoru Kumazaki 1,2, Toshiki Kishimoto', Arata Furukawa ${ }^{3}$, Hiroyuki Mori ${ }^{4}$, Yoshiki Tanaka ${ }^{3}$, \\ Naoshi Dohmae ${ }^{2}$, Ryuichiro Ishitani ${ }^{1,2}$, Tomoya Tsukazaki ${ }^{3,5}$ \& Osamu Nureki ${ }^{1,2}$
}

'Department of Biological Sciences, Graduate School of Science, The University of Tokyo, 7-3-1 Hongo, Bunkyo-ku, Tokyo 113 0033, Japan, ${ }^{2}$ Global Research Cluster, RIKEN, 2-1 Hirosawa, Wako-shi, Saitama 351-0198, Japan, ${ }^{3}$ Department of Systems Biology, Graduate School of Biological Sciences, Nara Institute of Science and Technology, 89 16-5 Takayama-cho, Ikoma, Nara 630-0192, Japan, ${ }^{4}$ Institute for Virus Research, Kyoto University, Shogoin Kawara-cho, Sakyo-ku, Kyoto 606-8507, Japan, ${ }^{5}$ JST, PRESTO, 4-1-8 Honcho, Kawaguchi, Saitama 332-0012, Japan.

Bacterial YidC, an evolutionally conserved membrane protein, functions as a membrane protein chaperone in cooperation with the Sec translocon and as an independent insertase for membrane proteins. In Gram-negative bacteria, the transmembrane and periplasmic regions of YidC interact with the Sec proteins, forming a multi-protein complex for Sec-dependent membrane protein integration. Here, we report the crystal structure of full-length Escherichia coli YidC. The structure reveals that a hydrophilic groove, formed by five transmembrane helices, is a conserved structural feature of YidC, as compared to the previous YidC structure from Bacillus halodurans, which lacks a periplasmic domain. Structural mapping of the substrateor Sec protein-contact sites suggested the importance of the groove for the YidC functions as a chaperone and an insertase, and provided structural insight into the multi-protein complex.

 he YidC/Oxa1/Alb3 family members are conserved in bacteria, mitochondria and chloroplasts, and are involved in the biogenesis of membrane proteins ${ }^{1}$, especially respiratory chain complexes. In bacteria, YidC facilitates the folding and assembly of membrane proteins inserted by a conserved protein-conducting channel, the Sec translocon, and also inserts proteins into the membrane independently ${ }^{2}$. YidC contains the five core transmembrane (TM) helices conserved among the family members. Recently, we reported the crystal structure of YidC from Bacillus halodurans (BhYidC) at $2.4 \AA$ resolution ${ }^{3}$. The structure revealed that the core TM helices form a hydrophilic groove that contains many conserved hydrophilic residues and is open toward both the lipid bilayer and the cytoplasm. Structure-based in vivo analyses revealed the interactions between the hydrophilic groove and a substrate protein, as well as the importance of an arginine residue in the groove for membrane protein insertion mediated by Bacillus subtilis YidC.

Escherichia coli YidC (EcYidC) is essential for cell viability ${ }^{4}$ and one of the best-studied YidC/Oxa1/Alb3 family members ${ }^{1}$. In E. coli, YidC associates with the SecYEG complex and the SecDFYajC complex ${ }^{5-7}$ to form the holotranslocon, a hetero-complex composed of single copies of SecYEG, SecDFYajC and YidC, which contributes to efficient membrane protein biogenesis in the Sec-dependent pathway ${ }^{7}$. As YidC is much more abundant than the SecYEG complex ${ }^{8}$, it is likely that only a portion of the YidC protein pool forms the holo-translocon. The YidC proteins from Gram-negative bacteria possess an additional TM helix, which functions as a signal sequence, and a large periplasmic domain (P1) that protrudes from the N-termini of the core TM helices. Although the P1 domain is not essential for the YidC function', it interacts with multiple components of the Sec machinery and is considered to facilitate stable complex formation ${ }^{6,10}$. Previous structural and biochemical analyses suggested assembly models of the YidC-Sec complex ${ }^{6,7}$, but the structural details, including the orientation of the P1 domain, still remain uncertain. A high-resolution structure of full-length EcYidC is required to understand the molecular mechanism underlying Sec-dependent membrane protein integration.

\section{Results}

Crystal structure of EcYidC. We crystallized EcYidC in the lipidic cubic phase, and determined the structure by molecular replacement, using the P1 domain of EcYidC (PDB ID: 3BS6) and the TM region of BhYidC (PDB ID: 
3WO6) as search models. The final model was refined at $3.2 \AA$ resolution to $R_{\text {work }}=22.1 \%$ and $R_{\text {free }}=27.7 \%$ (Table 1, Fig. $1 \mathrm{~A}$ and Supplementary Fig. S1). In the crystal lattice, the molecules appeared to exist as monomers, and did not form an effective oligomer as proposed previously (Supplementary Fig. S2) (11, $^{12}$, similar to the crystal structures of $\mathrm{BhYidC}^{3}$. This observation suggested that YidC functions as a monomer in the cytoplasmic membrane, consistent with the recent findings that monomeric EcYidC binds to translating ribosomes in a detergent solution and in lipid membranes ${ }^{13-15}$.

The EcYidC structure comprises five TM $\alpha$-helices (TM2-6) and the protruding $\mathrm{P} 1$ and $\mathrm{C} 1$ regions (Fig. $1 \mathrm{~A}$ ). The $\mathrm{P} 1$ domain is connected to the TM region by the $\mathrm{PH} 1$ helix, which is amphipathic and lies parallel to the plane of the membrane. The $\mathrm{C} 2$ region and the $\mathrm{C}$ terminal C3 tail are structurally disordered, as also found in the BhYidC structures ${ }^{3}$. The architecture of the core TM region of EcYidC is also similar to that of BhYidC ${ }^{3}$. The TM2 and TM3 helices are kinked at the conserved proline residues (Pro371 and Pro431, respectively) in the middle of the lipid bilayer. The five TM $\alpha$-helices are tightly packed together in the periplasmic leaflet (Fig. 1B), but loosely interact with each other in the cytoplasmic leaflet. This results in a hydrophilic groove, opened toward both the cytoplasmic and membrane sides through a gap between the TM3 and TM5 helices (Fig. 1C). The groove contains many hydrophilic residues, including Thr362, Arg366, Thr373, Gln429, Thr474, Ser520, Asn521 and Gln527 (Fig. 1D). In particular, the highly conserved Arg366 generates a positively-charged surface in the groove (Fig. $1 \mathrm{C}$ and Supplementary Fig. S3).

The YidC proteins from Gram-negative bacteria have the TM1 helix and the P1 domain, in addition to the core TM region. In the EcYidC structure, the TM1 helix and the following N-terminal region of the $\mathrm{P} 1$ domain were disordered, although $\mathrm{N}$-terminal sequencing confirmed that these TM1 helix residues were intact in

Table 1 | Data collection and refinement statistics

EcYidC

PDB ID code

3WVF

\begin{tabular}{|c|c|}
\hline Data Collection & \\
\hline Space group & $P 1$ \\
\hline Cell dimensions & \\
\hline$a, b, c(\AA)$ & $42.7,76.0,91.3$ \\
\hline$\alpha, \beta, \gamma()^{\circ}$ & $78.1,82.4,77.6$ \\
\hline Resolution $(\AA)^{\text {a }}$ & $50.0-3.20(3.26-3.20)$ \\
\hline$R_{\text {sym }}$ a b & $0.188(0.369)$ \\
\hline$l / \sigma l^{\mathrm{a}}$ & $6.19(2.44)$ \\
\hline Completeness (\%) ${ }^{a}$ & $91.4(89.3)$ \\
\hline Redundancy ${ }^{a}$ & $2.8(2.3)$ \\
\hline Refinement & \\
\hline Resolution $(\AA)^{a}$ & $50.0-3.20(3.29-3.20)$ \\
\hline No. reflections & 16,470 \\
\hline$R_{\text {work }} / R_{\text {free }}{ }^{c}$ & $22.1 / 27.7$ \\
\hline No. atoms (Total) & 6,943 \\
\hline No. atoms (Protein) & 6,943 \\
\hline B-factors (Protein) & 34.00 \\
\hline Root mean square deviations & \\
\hline Bond lengths $(A)$ & 0.003 \\
\hline Bond angles $\left(1^{\circ}\right)$ & 0.770 \\
\hline Ramachandran plot & \\
\hline Favored (\%) & 96.9 \\
\hline Allowed (\%) & 3.1 \\
\hline Outliers (\%) & 0.0 \\
\hline 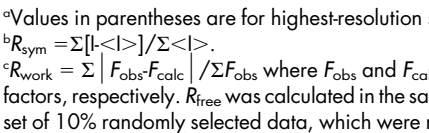 & $\begin{array}{l}\text { the observed and calculated structure } \\
\text { vay as } R_{\text {work }} \text { using reflections belong to a tes } \\
\text { sed to fit the model. }\end{array}$ \\
\hline
\end{tabular}

the purified YidC. This suggested that the TM1 helix in the YidC proteins from Gram-negative bacteria does not specifically interact with the other TM helices, consistent with the fact that the helix is not important for the YidC activity ${ }^{9}$.

The P1 domain consists of two anti-parallel $\beta$-sheets, which form a tightly packed $\beta$-supersandwich fold. The P1 domain of the fulllength EcYidC structure is essentially identical to the reported crystal structures of the isolated P1 domains (PDB ID 3BS6, rmsd $=0.660$ and PDB ID 3BLC, rmsd $=0.672$, over the $\mathrm{C} \alpha$ atoms of residues 59 to $320)^{16,17}$. The P1 domain is connected to the PH1 helix by a short loop. Thus, the P1 domain is likely to be located on the extracellular surface of the membrane, which is consistent with a previous report that the P1 domain alone interacts with $E$. coli membrane lipids ${ }^{17}$. In addition, the P1 domain forms several hydrogen bonds with the P2 region, the loop connecting the TM3 and TM4 helices, and the TM3 helix. The main-chain carbonyl groups of Pro88 and Met441 hydrogen bond with the guanidinium group of Arg447 and the side-chain amide group of Gln258 and the side-chain hydroxyl group of Tyr259, respectively (Fig. 1B). These interaction sites are highly conserved in the YidC proteins from Gram-negative bacteria (Fig. 1E). Taken together, these observations suggest that the membrane binding and several hydrogen bonds stabilize the orientation of the P1 domain of YidC.

Characteristics of the YidC structure. A comparison of the EcYidC and BhYidC structures revealed a difference in the $\mathrm{Cl}$ region (Fig. 2A, B). The arrangement of two antiparallel helices in the $\mathrm{C} 1$ region of EcYidC is rotated by $35^{\circ}$ with respect to the core region, as compared with the BhYidC structure. In the crystal, the $\mathrm{C} 1$ region contacts the P1 domains of the adjacent molecules (Supplementary Fig. S2). A short loop between the $\mathrm{CH} 1$ and $\mathrm{CH} 2$ helices interacts with the $\beta 7$ strand and a loop between the $\beta 6$ strand and the $\eta 1$ helix. The CH1 helix interacts with the $\eta 1$ helix. Despite these crystal contacts, the crystallographic B-factors of the $\mathrm{C} 1$ region are higher than those of the other regions in the EcYidC structure (Fig. 2C). Considering our previous results showing the importance of the flexible $\mathrm{C} 1$ region of $\mathrm{BhYidC}^{3}$, the structural difference of the $\mathrm{C} 1$ region and the higher crystallographic $\mathrm{B}$-factors suggested that the flexibility of the $\mathrm{C} 1$ region is a universally conserved feature of YidC.

By contrast, the conserved hydrophilic residues in EcYidC, and especially the highly conserved arginine residue (Arg366) in the hydrophilic groove (Fig. 1D and Supplementary Fig. S3), were located at exactly the same places as those in BhYidC, suggesting that the arginine residue is important for the EcYidC function, as found for BhYidC ${ }^{3}$.

Positively-charged residue in the groove. To assess the importance of the highly conserved arginine residue, we performed a growth complementation assay using a YidC-depletable strain, JS7131 ${ }^{4}$, in which YidC expression is controlled by the arabinose promoter. In this assay, the YidC mutants were expressed under the control of the lac promoter from the pTV118N-based plasmids. All of the mutants with mutations in the hydrophilic groove, except for the reportedlyinactive $\mathrm{T} 362 \mathrm{~A}$ mutant ${ }^{15}$, rescued lethal phenotype at $37^{\circ} \mathrm{C}$ in the absence of arabinose (Fig. 3A), which is consistent with recent studies $^{15,18}$. In contrast, the R366A and R366M mutants could not rescue the lethal phenotype at $20^{\circ} \mathrm{C}$, whereas the $\mathrm{R} 366 \mathrm{~K}$ mutant could (Fig. 3B). These results indicated that a positively-charged residue, an arginine or a lysine, at position 366 is not essential, but play an important role for the YidC function under certain conditions. A positively-charged residue at this position is conserved among the YidC/Oxa1/Alb3 family members (Supplementary Fig. S3). The corresponding mutation of R366A in yeast Oxa1 (R140A) also led to a cold-sensitive phenotype ${ }^{19}$ (Supplementary Fig. S3). Altogether, we concluded that the YidC/Oxa1/Alb3 family members possess the evolutionarily conserved positively charged groove, which are related to their functions. 
A

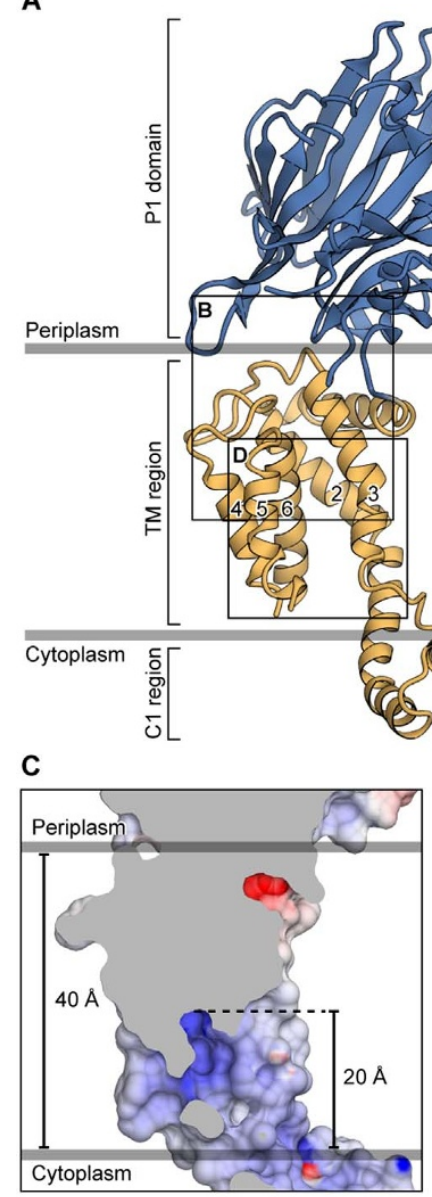

B

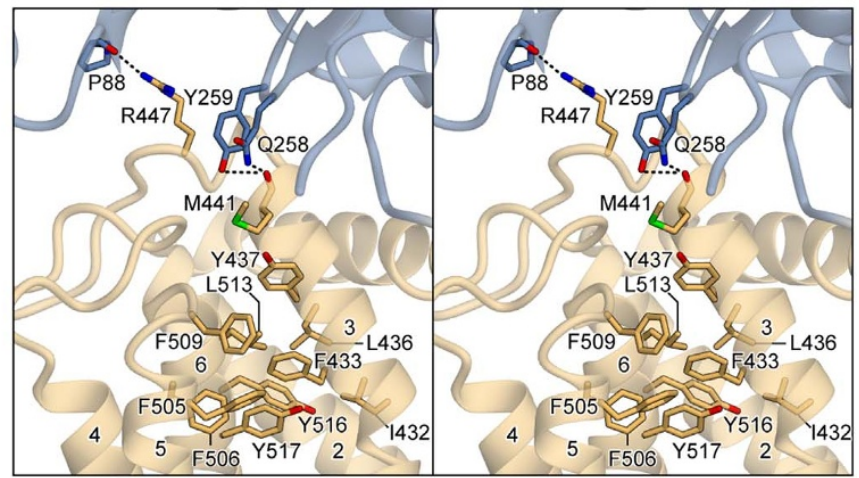

$\mathrm{E}$

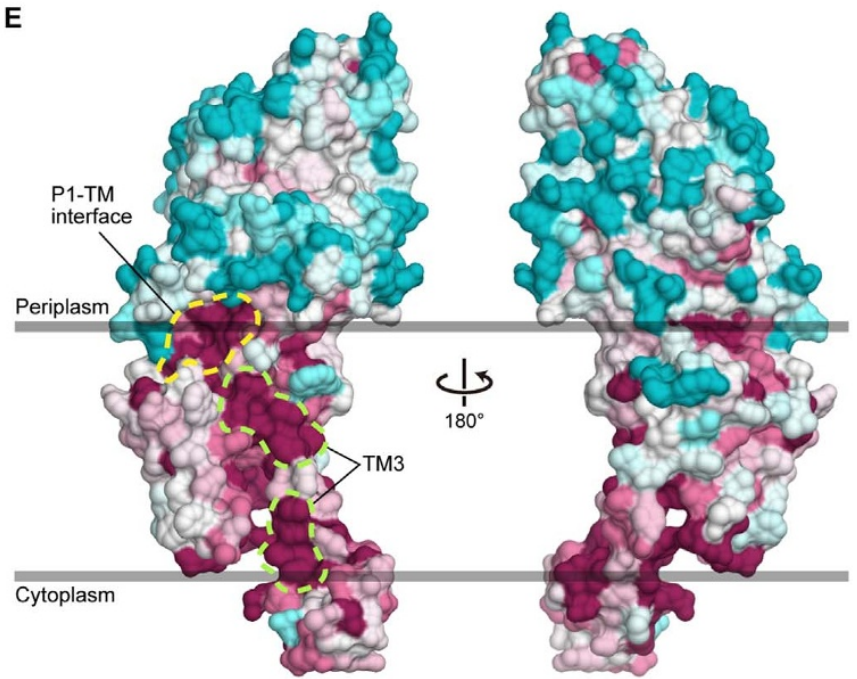

Figure 1 Structure of EcYidC. (A), Ribbon representations of the EcYidC structure, viewed transversely through the membrane. (B), Close-up view of the hydrophobic core of EcYidC and the interaction surface between the P1 domain and the TM region (stereo view). Hydrogen bonds are shown as dashed lines. (C), Surface model representations of EcYidC, sliced through the center of the groove. The structure is colored according to the electrostatic potential, ranging from blue $(+10 \mathrm{kT} / \mathrm{e})$ to red $(-10 \mathrm{kT} / \mathrm{e})$. (D), Close-up view of the hydrophilic groove of EcYidC (colored), showing hydrophilic residues. BhYidC (grey) is superimposed on the TM region of EcYidC. The side chain of Arg72 in BhYidC (corresponding to Arg366 in EcYidC) is shown. (E), Surface model representations of EcYidC colored according to sequence conservation, ranging from cyan (low) to maroon (high). Dotted regions highlight the conserved surfaces in the P1-TM interface (yellow) and the TM3 helix (light green).

Substrate-contacting sites. A number of substrate-contacting residues were reported for EcYidC and BhYidC, as identified by cross-linking analyses with Sec-independent substrates, the Pf3 coat protein ${ }^{20}, \mathrm{~F}_{\mathrm{o}} \mathrm{c}^{21}$, and $\mathrm{MifM}^{3}$. These residues mapped mainly to one face of the exterior surface of the TM region, as well as the interior surface of the hydrophilic groove (Fig. 4A, B), revealing that the substrate-contacting residues in EcYidC are clustered in the vicinity of the groove opening toward the membrane in the TM3 and TM5 helices. These observations highlighted the importance of both the hydrophobic and hydrophilic interactions ${ }^{3}$ between substrates and YidC for the Sec-independent insertion. Moreover, the contact sites with a Sec-dependent substrate, FtsQ, share the same regions with the Sec-independent substrates ${ }^{21}$ (Fig. 4C), supporting the notion that the hydrophilic groove and the TM helices adjacent to the groove opening are also important for the function of EcYidC, as a membrane protein chaperone in Secdependent membrane protein integration.

\section{Discussion}

We have presented the crystal structure of full-length EcYidC, which reveals the orientation of the $\mathrm{P} 1$ domain with respect to the TM segments and allows accurate mapping of the previous crosslinking results. The structure also shows that a hydrophilic groove formed by the TM helices is a common structural feature among the YidC proteins.
The P1 domain shares structural similarity with galactose mutarotase, and possesses a large cleft located at the position corresponding to its sugar binding pocket, although the domain lacks a sugar binding motif ${ }^{16,17}$. This cleft has been proposed to be a binding site for substrate proteins or periplasmic molecules ${ }^{16,17}$. Our crystal structure revealed that the cleft is oriented away from the membrane (Fig. 5), suggesting that the cleft could bind periplasmic molecules or proteins, rather than substrate proteins. However, further biological experiments are needed to clarify the function of the P1 domain in vivo.

The holo-translocon, composed of single copies of YidC, SecYEG and SecDFYajC, is necessary for effective protein translocation and integration $^{5,7}$. Our EcYidC structure and previous studies ${ }^{6,10,22}$ have provided insights into the structural organization of the holo-translocon. SecF interacts with part of the P1 domain of EcYidC (residues 215-265), in which one residue (Lys249) was cross-linked with SecG, $\mathrm{SecD}$ and $\mathrm{YajC}^{6}$ (Fig. 5), suggesting that this surface provides the interface between YidC and the SecYEG-SecDFYajC complex. The highly conserved surface formed by the TM3 helices, which are located on the lateral edge of the hydrophilic groove, may provide interaction sites for the TM regions of the Sec proteins (Fig. 1E). Indeed, given that YidC was cross-linked with the lateral gate of $\operatorname{Sec} \mathrm{Y}^{6}$, and that nascent TM segments of the substrate sequentially interact with SecY and YidC, it is likely that the lateral gate of SecY 


\section{A}

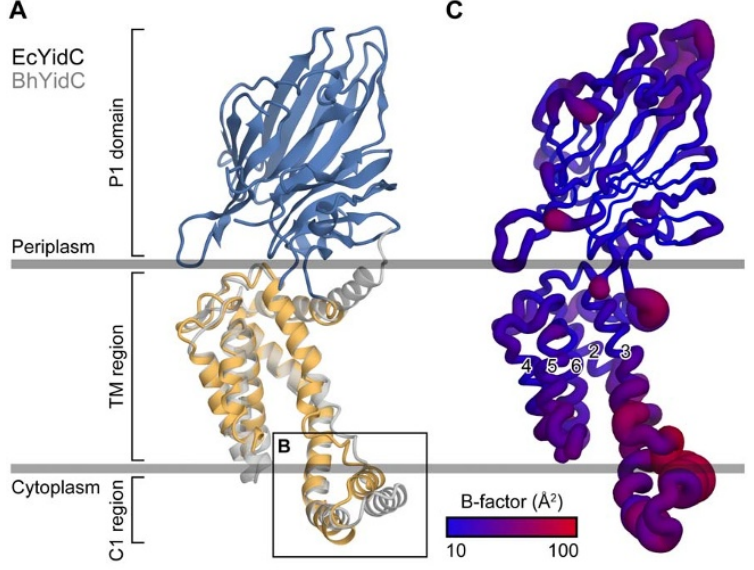

B

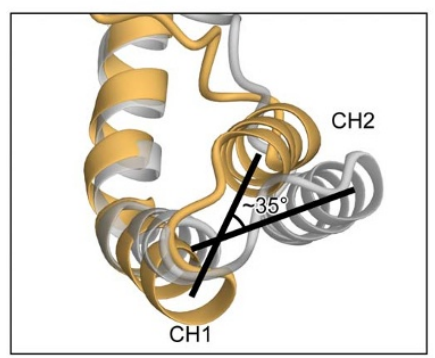

Figure $2 \mid$ Flexibility of the $\mathrm{C} 1$ region. (A), Superimposition of the crystal structures of EcYidC (colored) and BhYidC (PDB ID: 3WO6, grey). (B), Close-up view of the $\mathrm{C} 1$ region. The arrangement of the helices in the $\mathrm{C} 1$ region is rotated by $\sim 35^{\circ}$, as compared with those in BhYidC, with respect to the core region. $(\mathrm{C})$, The crystallographic B-factors are colored in a gradient ranging from blue $\left(10 \AA^{2}\right)$ to red $\left(100 \AA^{2}\right)$.
A

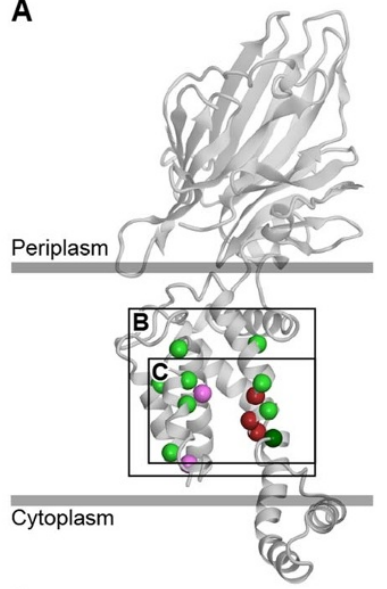

C

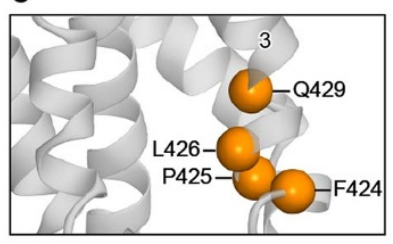

B

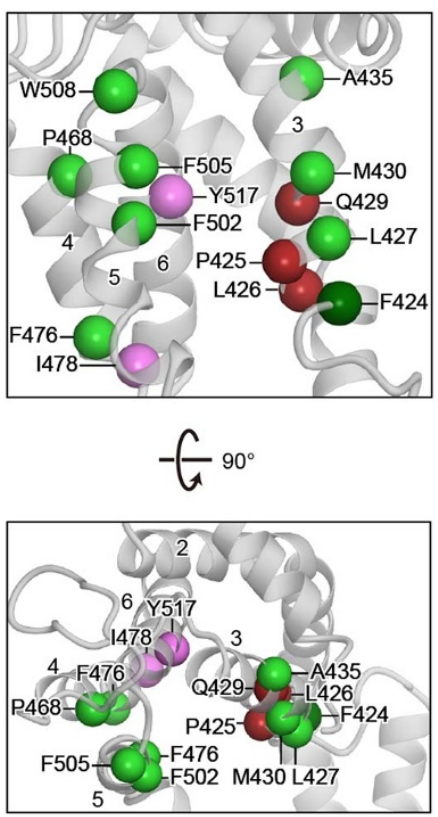

Figure $4 \mid$ Structural mapping of substrate-contacting residues. (A), Mapping of the substrate-contacting residues. (B), (C), Close-up views of the boxed areas in A. The residues bound to Sec-independent substrates are shown as spheres, colored green (the Pf3 coat protein), pink (MifM), brown $\left(\mathrm{F}_{\mathrm{o}} \mathrm{c}\right)$ and dark green (both the Pf3 coat protein and $\mathrm{F}_{\mathrm{o}} \mathrm{c}$ ), and viewed from the membrane (upper) and periplasmic (lower) sides (B). The residues bound to the $\mathrm{Sec}$-independent substrate, FtsQ, are shown as orange spheres (C). The P1 domain was removed for clarity.
A

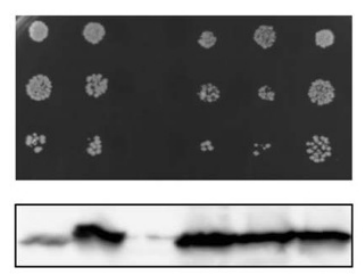

$37^{\circ} \mathrm{C}$

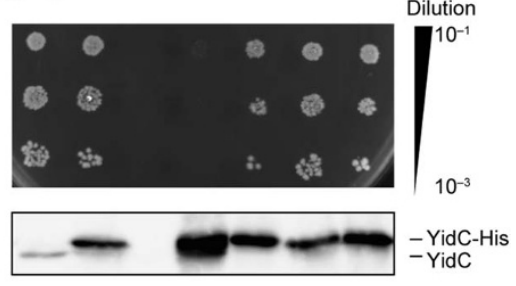

B

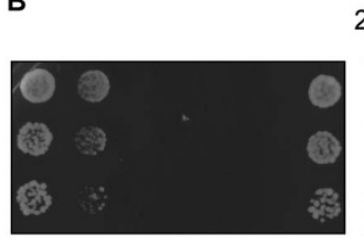

$20^{\circ} \mathrm{C}$
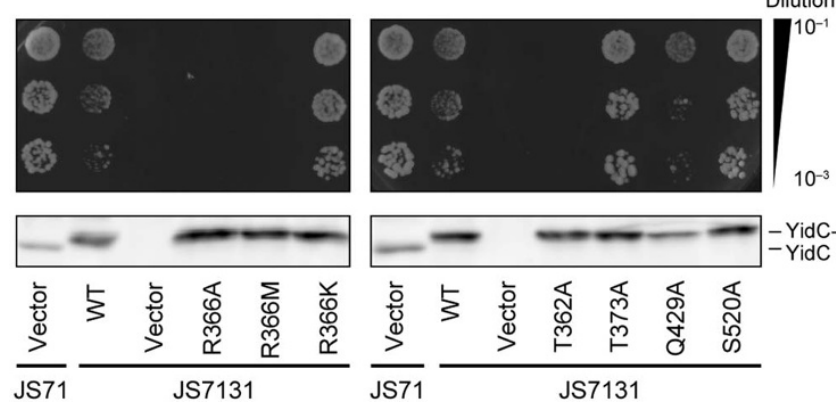

Figure 3 Growth complementation of EcYidC mutants. (A), (B), Growth complementation of JS7131 ( $\Delta$ yidC, $\left.\mathrm{P}_{\text {ara }}-y i d C\right)^{4}$ by EcYidC mutants under conditions of wild-type EcYidC depletion ( $0.4 \%$ glucose $)$ at $37^{\circ} \mathrm{C}$ (A) and $20^{\circ} \mathrm{C}(\mathrm{B})$. The cellular accumulation of the mutants was detected by immunoblotting, using anti-YidC antibody. The strain JS71 $\left(y i d C^{+}, \mathrm{P}_{\text {ara }}-y i d C\right)^{4}$ possesses two functional yidC genes.
A

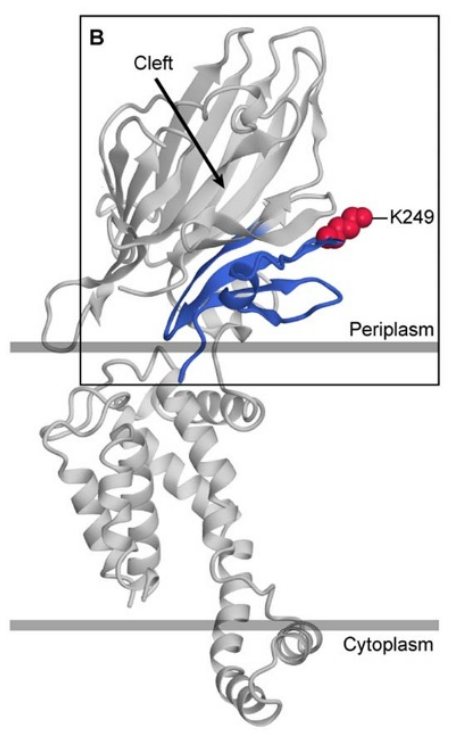

B
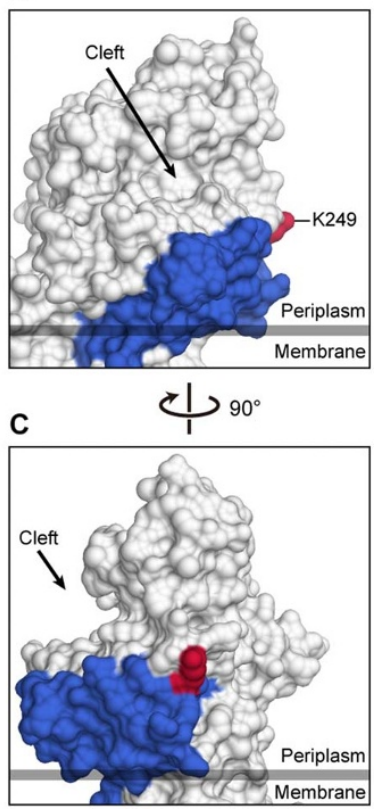

Figure 5 Structural insight into the role of the P1 domain. (A), The regions interacting with SecF are colored blue, with Lys249 highlighted in red. (B), (C), Surface model representations of the boxed areas of EcYidC in A (B), and viewed from the other side (C). The cleft in the P1 domain is indicated by arrows. 
faces the hydrophilic groove of YidC in Sec-dependent membrane protein integration. This complex formation may facilitate the folding of multi-spanning membrane proteins, such as $\mathrm{LacY}^{23}$. In this process, YidC could form a transient complex with the nascent TM segment, due to hydrophilic interactions between the charged or polar residues of the substrate and the hydrophilic groove of YidC in the lipid bilayer, until an assembly partner emerges from the Sec translocon. Chaperones for cytosolic proteins prevent non-specific intramolecular hydrophobic interactions in the substrates, whereas YidC can provide a hydrophilic binding surface for immature membrane proteins to accomplish proper folding. Therefore, the hydrophilic groove may be important for the chaperone activity of YidC, as well as for its insertase activity.

\section{Methods}

Purification and crystallization of EcYidC. E. coli yidc, from JCM20135 genomic DNA (provided by RIKEN), was cloned into a modified pTV118N (TAKARA) vector. The resulting plasmid, encoding EcYidC $_{1-540}$-LESSGENLYFQGQFTS- $\mathrm{H}_{8}$, was introduced into E. coli strain BL21(DE3). The cells were cultivated at $37^{\circ} \mathrm{C}$ in a 10 1 culture, supplemented with $50 \mu \mathrm{g} \mathrm{ml}^{-1}$ ampicillin, to an absorbance at $600 \mathrm{~nm}$ of $\sim 0.6$, induced with $1 \mathrm{mM}$ isopropyl- $\beta$-D-thiogalactopyranoside for $18 \mathrm{~h}$, and harvested by centrifugation $\left(4,500 \mathrm{~g}, 10 \mathrm{~min}, 4^{\circ} \mathrm{C}\right)$. Total membranes were prepared as described previously ${ }^{3}$. EcYidC was solubilized for $1 \mathrm{~h}$ at $4^{\circ} \mathrm{C}$, in a buffer containing $20 \mathrm{mM} \mathrm{N}$-(2-acetamido)iminodiacetic acid (ADA)- $\mathrm{NaOH}, \mathrm{pH} 5.6,300 \mathrm{mM} \mathrm{NaCl}$, $2 \% \mathrm{n}$-decyl- $\beta$-D-maltoside (DM) and $0.1 \mathrm{mM}$ phenylmethylsulfonyl fluoride. After ultracentrifugation $\left(138,000 \mathrm{~g}, 30 \mathrm{~min}, 4^{\circ} \mathrm{C}\right)$, the supernatant was mixed with $10 \mathrm{ml}$ Ni Sepharose excel (GE Healthcare), equilibrated with buffer A (20 mM ADA-

$\mathrm{NaOH}, \mathrm{pH} 5.6,300 \mathrm{mM} \mathrm{NaCl}$ and $0.25 \% \mathrm{DM}$ ) containing $30 \mathrm{mM}$ imidazole- $\mathrm{HCl}$, $\mathrm{pH} 6.6$, for $30 \mathrm{~min}$ at $4^{\circ} \mathrm{C}$. The resin was washed with buffer A containing $50 \mathrm{mM}$ imidazole- $\mathrm{HCl}, \mathrm{pH} 6.6$, and then EcYidC was eluted with a gradient of 100-500 mM imidazole-HCl, pH 6.6, in buffer A. The eluate was mixed with the same volume of a buffer containing $500 \mathrm{mM}$ ADA- $\mathrm{NaOH}, \mathrm{pH} 5.6,300 \mathrm{mM} \mathrm{NaCl}$ and $0.25 \% \mathrm{DM}$, and then repeatedly concentrated and diluted with buffer A, using an Amicon Ultra filter (50 kDa molecular weight cutoff, Merck Millipore), to remove the imidazole. The Cterminal $\mathrm{His}_{8}$-tag was cleaved by His-tagged TEV protease (laboratory stock) in buffer A supplemented with $10 \mathrm{mM}$ imidazole- $\mathrm{HCl}, \mathrm{pH} 6.6$, for $12 \mathrm{~h}$ at $4^{\circ} \mathrm{C}$. The sample was loaded on a $10 \mathrm{ml} \mathrm{Ni}$ Sepharose excel column, equilibrated with buffer A supplemented with $10 \mathrm{mM}$ imidazole- $\mathrm{HCl}$, and the flow-through was concentrated and loaded on a HiLoad 16/600 Superdex 200 column (GE Healthcare), which was eluted with buffer A. The N-terminal five amino acid sequence of the purified YidC, Met-Asp-Ser-Gln-Arg, was confirmed by Edman sequencing with a PPSQ-31A protein sequencer (SHIMADZU). For crystallization, the purified EcYidC was concentrated to approximately $15 \mathrm{mg} \mathrm{ml}^{-1}$. The crystals of EcYidC appeared from the mixture of the protein and monoolein in a reservoir solution containing $25 \%$ poly(ethyleneglycol) $600,100 \mathrm{mM}$ MES- $\mathrm{NaOH}, \mathrm{pH}$ 5.9, $50 \mathrm{mM}$ potassium thiocyanate and $12 \mathrm{mM}$ triglycine, prepared by the twin-syringe mixing and crystallization method described previously ${ }^{3}$. The crystals grew to dimensions of $50 \times$ $10 \times 5 \mu \mathrm{m}$ in 2 weeks, and were then flash-cooled and stored in liquid nitrogen

Data collection and structure determination. The X-ray diffraction data were collected at SPring- 8 beamline BL32XU ${ }^{24}$ and processed using HKL2000 (HKL Research Inc.). Initial phases were calculated by molecular replacement with the crystal structure of the P1 domain of EcYidC (PDB ID: 3BS6) using PHASER ${ }^{25}$, which provided a partial model and weak electron density maps for the TM region of EcYidC. However, the electron density map was not sufficiently interpretable to build the model. To improve the initial phases, molecular replacement was performed using the P1 domain of EcYidC and the TM region of BhYidC (PDB ID: 3WO6) ${ }^{3}$ as search models. The model was manually rebuilt using COOT $^{26}$ and refined using PHENIX ${ }^{27}$. To reduce the model bias and improve the phases, simulated annealing refinement was performed at an early stage of the model building, followed by several cycles of positional refinement combined with individual B-factor refinement. The final stage of refinement was performed by using X-ray/stereochemistry weight and X-ray/ADP weight optimization. Finally, the structure of EcYidC was refined to $R_{\text {work }}=22.1 \%$ and $R_{\text {free }}=27.7 \%$ at $3.2 \AA$ resolution. The refinement statistics are summarized in Table 1. The Ramachandran plots were calculated with RAMPAGE ${ }^{28}$. The crystal contains two molecules in the asymmetric unit ( $\mathrm{Mol} \mathrm{A}$ and $\mathrm{Mol} \mathrm{B}$ ). The overall structures of Mol A and Mol B are almost identical (root-mean-square deviation, $0.884 \AA$ over the $\mathrm{C} \alpha$ atoms of residues $56-528$ ). The atomic coordinates and structure factors have been deposited in the Protein Data Bank, under the accession code $3 \mathrm{WVF}$. The molecular graphics were illustrated with CueMol (http://www.cuemol. org/). The sequence conservation among 218 EcYidC orthologs, which share at least $30 \%$ amino acid sequence identity with EcYidC, was colored on the surface of EcYidC using the ConSurf server (http://consurf.tau.ac.il/). Electrostatic potentials were calculated using $\mathrm{APBS}^{29}$

Complementation test of EcYidC mutants. Mutations were introduced into the EcYidC-expression plasmid by site-directed mutagenesis. To adjust the accumulation of YidC, we additionally mutated the initial codon, ATG, to $\mathrm{GTG}^{30}$. The resultant plasmid, encoding the mutant V-EcYidC 2 -548-LESSGENLYFQGQFTS- $\mathrm{H}_{8}$, was introduced into the JS7131 strain ${ }^{4}$. The E. coli cells were cultured at $37^{\circ} \mathrm{C}$ in LB medium supplemented with $0.2 \%$ arabinose and $50 \mu \mathrm{g} \mathrm{ml}^{-1}$ ampicillin until they attained an $\mathrm{A}_{600}$ of 0.4 , diluted, spotted onto LB-agar plates supplemented with $0.4 \%$ glucose and $50 \mu \mathrm{g} \mathrm{ml}^{-1}$ ampicillin, and incubated at $37^{\circ} \mathrm{C}$ for $24 \mathrm{~h}$ or at $20^{\circ} \mathrm{C}$ for $\sim 120 \mathrm{~h}$. To determine the accumulation level of YidC at $20^{\circ} \mathrm{C}$, the preculture cells, grown in LB medium supplied with $0.2 \%$ arabinose and $50 \mu \mathrm{g} \mathrm{ml}^{-1}$ ampicillin at $37^{\circ} \mathrm{C}$, were cultured in LB medium supplied with $0.4 \%$ glucose and $50 \mu \mathrm{g} \mathrm{ml}^{-1}$ ampicillin for $12 \mathrm{~h}$ at $37^{\circ} \mathrm{C}$ or for $12 \mathrm{~h}$ at $20^{\circ} \mathrm{C}$. EcYidC accumulation was detected by immunoblotting using anti-YidC antiserum, raised in a rabbit immunized with the synthetic peptide $\left(\mathrm{NH}_{2}-\mathrm{C}\right.$-TPDEKYEKYKFDTIADN-COOH), corresponding to amino acids 225-241 of EcYidC.

1. Saller, M. J., Wu, Z. C., de Keyzer, J. \& Driessen, A. J. M. The YidC/Oxa1/Alb3 protein family: common principles and distinct features. Biol. Chem. 393, 1279-90 (2012).

2. Dalbey, R. E., Kuhn, A., Zhu, L. \& Kiefer, D. The membrane insertase YidC. Biochim. Biophys. Acta 1843, 1489-96 (2014).

3. Kumazaki, K. et al. Structural basis of Sec-independent membrane protein insertion by YidC. Nature 509, 516-20 (2014).

4. Samuelson, J. C. et al. YidC mediates membrane protein insertion in bacteria. Nature 406, 637-41 (2000).

5. Nouwen, N. \& Driessen, A. J. M. SecDFyajC forms a heterotetrameric complex with YidC. Mol. Microbiol. 44, 1397-1405 (2002).

6. Sachelaru, I. et al. YidC occupies the lateral gate of the SecYEG translocon and is sequentially displaced by a nascent membrane protein. J. Biol. Chem. 288, 1-26 (2013).

7. Schulze, R. J. et al. Membrane protein insertion and proton-motive-forcedependent secretion through the bacterial holo-translocon SecYEG-SecDF-YajCYidC. Proc. Natl. Acad. Sci. U. S. A. 111, 4844-9 (2014).

8. Urbanus, M. L. et al. Targeting, insertion, and localization of Escherichia coli YidC. J. Biol. Chem. 277, 12718-23 (2002).

9. Jiang, F. et al. Defining the regions of Escherichia coli YidC that contribute to activity. J. Biol. Chem. 278, 48965-72 (2003).

10. Xie, K., Kiefer, D., Nagler, G., Dalbey, R. E. \& Kuhn, A. Different regions of the nonconserved large periplasmic domain of Escherichia coli YidC are involved in the SecF interaction and membrane insertase activity. Biochemistry 45, 13401-8 (2006).

11. Lotz, M., Haase, W., Kühlbrandt, W. \& Collinson, I. Projection structure of yidC: a conserved mediator of membrane protein assembly. J. Mol. Biol. 375, 901-7 (2008).

12. Kohler, R. et al. YidC and Oxal form dimeric insertion pores on the translating ribosome. Mol. Cell 34, 344-53 (2009).

13. Kedrov, A. et al. Elucidating the native architecture of the YidC: ribosome complex. J. Mol. Biol. 425, 4112-24 (2013).

14. Seitl, I., Wickles, S., Beckmann, R., Kuhn, A. \& Kiefer, D. The C-terminal regions of YidC from Rhodopirellula baltica and Oceanicaulis alexandrii bind to ribosomes and partially substitute for SRP receptor function in Escherichia coli. Mol. Microbiol. 91, 408-21 (2013).

15. Wickles, S. et al. A structural model of the active ribosome-bound membrane protein insertase YidC. Elife 3, e03035 (2014).

16. Oliver, D. C. \& Paetzel, M. Crystal structure of the major periplasmic domain of the bacterial membrane protein assembly facilitator YidC. J. Biol. Chem. 283 , 5208-5216 (2008).

17. Ravaud, S., Stjepanovic, G., Wild, K. \& Sinning, I. The crystal structure of the periplasmic domain of the Escherichia coli membrane protein insertase YidC contains a substrate binding cleft. J. Biol. Chem. 283, 9350-8 (2008).

18. Chen, Y., Soman, R., Shanmugam, S. K., Kuhn, A. \& Dalbey, R. E. The role of the strictly conserved positively charged residue differs among the Gram-positive, Gram-negative and chloroplast YidC homologs. J. Biol. Chem. (2014) doi:10.1074/jbc.M114.595082.

19. Lemaire, C., Guibet-Grandmougin, F., Angles, D., Dujardin, G. \& Bonnefoy, N. A yeast mitochondrial membrane methyltransferase-like protein can compensate for oxal mutations. J. Biol. Chem. 279, 47464-72 (2004).

20. Klenner, C. \& Kuhn, A. Dynamic Disulfide Scanning of the Membrane-inserting Pf3 Coat Protein Reveals Multiple YidC Substrate Contacts. J. Biol. Chem. 287, 3769-76 (2012).

21. Yu, Z., Koningstein, G., Pop, A. \& Luirink, J. The conserved third transmembrane segment of YidC contacts nascent Escherichia coli inner membrane proteins. J. Biol. Chem. 283, 34635-42 (2008).

22. Urbanus, M. L. et al. Sec-dependent membrane protein insertion: sequential interaction of nascent FtsQ with SecY and YidC. EMBO Rep. 2, 524-529 (2001).

23. Nagamori, S., Smirnova, I. N. \& Kaback, H. R. Role of YidC in folding of polytopic membrane proteins. J. Cell Biol. 165, 53-62 (2004).

24. Hirata, K. et al. Achievement of protein micro-crystallography at SPring-8 beamline BL32XU. J. Phys. Conf. Ser. 425, 012002 (2013).

25. McCoy, A. J. et al. Phaser crystallographic software. J. Appl. Crystallogr. 40, 658-674 (2007)

26. Emsley, P., Lohkamp, B., Scott, W. G. \& Cowtan, K. Features and development of Coot. Acta Crystallogr. D. Biol. Crystallogr. 66, 486-501 (2010). 
27. Adams, P. D. et al. PHENIX: a comprehensive Python-based system for macromolecular structure solution. Acta Crystallogr. D. Biol. Crystallogr. 66, 213-21 (2010).

28. Lovell, S. C. et al. Structure validation by Calpha geometry: phi,psi and Cbeta deviation. Proteins 50, 437-50 (2003).

29. Baker, N. A., Sept, D., Joseph, S., Holst, M. J. \& McCammon, J. A. Electrostatics of nanosystems: application to microtubules and the ribosome. Proc. Natl. Acad. Sci. U. S. A. 98, 10037-41 (2001).

30. Park, E. \& Rapoport, T. A. Bacterial protein translocation requires only one copy of the SecY complex in vivo. J. Cell Biol. 198, 881-93 (2012).

\section{Acknowledgments}

We thank T. Nishizawa, H. Nishimasu, M. Hattori and R. Ishii for useful suggestions, A Kurabayashi and H. Nakamura for technical support, R. Yamazaki for secretarial assistance, the RIKEN BioResource Center (Ibaraki, Japan) for providing Escherichia coli genomic DNA, and the beamline staff members at BL32XU of SPring-8 (Hyogo, Japan) for technical assistance during data collection. The synchrotron radiation experiments were performed at BL32XU of SPring-8 (proposal Nos. 2012B1146, 2012B1162, 2013A1128, 2013A1168), with approval from RIKEN. This work was supported by the Platform for Drug Discovery, Informatics, and Structural Life Science from the Ministry of Education, Culture, Sports, Science and Technology (MEXT), by JSPS/MEXT KAKENHI (Grant Nos. 26119007, 26102532, 26291023, 25116705, 24227004, 13J08353), by the FIRST program, by PRESTO, JST, by a Grant-in-Aid for JSPS Fellows, by a grant for HPCI STRATEGIC PROGRAM Computational Life Science and Application in Drug Discovery and Medical Development from MEXT, by Astellas Foundation for Research on Metabolic Disorders, and by a Grant for Basic Science Research Projects from The Sumitomo Foundation.

\section{Author contributions}

K.K., T.K. and T.T. performed the crystallization and structure determination. A.F., Y.T. and H.M. performed the functional analysis. N.D. performed N-terminal sequencing. R.I and O.N. assisted with the structure determination. K.K., T.T. and O.N. wrote the manuscript. T.T. and O.N. directed and supervised all of the research.

\section{Additional information}

Accession codes The atomic coordinates and structure factors have been deposited in the Protein Data Bank, under the accession code $3 \mathrm{WVF}$.

Supplementary information accompanies this paper at http://www.nature.com/ scientificreports

Competing financial interests: The authors declare no competing financial interests.

How to cite this article: Kumazaki, K. et al. Crystal structure of Escherichia coli YidC, a membrane protein chaperone and insertase. Sci. Rep. 4, 7299; DOI:10.1038/srep07299 (2014).

This work is licensed under a Creative Commons Attribution-NonCommercialNoDerivs 4.0 International License. The images or other third party material in this article are included in the article's Creative Commons license, unless indicated otherwise in the credit line; if the material is not included under the Creative Commons license, users will need to obtain permission from the license holder in order to reproduce the material. To view a copy of this license, visit http:// creativecommons.org/licenses/by-nc-nd/4.0/ 